\title{
Estrategia para evaluar la competencia transversal "Aprendizaje Permanente" en la asignatura Transductores e Instrumentación Acústica
}

\author{
Jesús Alba Fernández ${ }^{a}$ y Romina del Rey Tormos ${ }^{a}$ \\ ${ }^{a}$ Universitat Politècnica de Valéncia, Escuela Politécnica Superior de Gandia, Departamento de Física \\ Aplicada, C/Paraninfo n ${ }^{\circ} 1$ - 46715 Grao de Gandia. jesalba@fis.upv.es, roderey@doctor.upv.es
}

\begin{abstract}
Use the learning in a strategically, autonomously and flexibly way, throughout life, according to the pursued objective is one of the transversal competences that is control item of the subject entitled "Transducers and Acoustic Instrumentation". This subject belong to the Bachelor's Degree in Engineering of Telecommunications Systems, Sound and Image. During the 2015-2016 academic year, we have initiated and presented strategies for start up the mechanisms in order to work and evaluate this transversal competence. We have defined a number of improvements for the 2016-2017 academic year. These improvements have based on the results obtained during the 2015-2016 academic year. This paper shows the evolution and valuation of the last two academic years
\end{abstract}

Keywords: transversal competence, lifelong learning, rubrics, the minute test, assessment, learning outcomes.

\section{Resumen}

Utilizar el aprendizaje de manera estratégica, autónoma y flexible, a lo largo de toda la vida, en función del objetivo perseguido es una de las competencias transversales que es punto de control de la asignatura "Transductores e Instrumentación Acústica" de la titulación del Grado en Ingeniería de Sistemas de Telecomunicación, Sonido e Imagen. En el curso 2015-2016 se iniciaron y presentaron estrategias de puesta en marcha de mecanismos para trabajarla en la asignatura y evaluarla. En base a los resultados obtenidos se definieron una serie de mejoras para el curso 20162017. En este trabajo se muestra la evolución y valoración de los dos cursos.

Palabras clave: competencia transversal, aprendizaje permanente, rúbricas, prueba del minuto, evaluación, resultados del aprendizaje. 


\section{Introducción}

Una definición de consenso de "competencia transversal" hace referencia a aquella habilidad relacionada con el desarrollo personal, que no depende de un ámbito temático o disciplinario especifico sino que aparece en todos los dominios de la actuación profesional y académica (González y Wagenaar, 2003). Estas habilidades deben utilizarlas los alumnos en base a diferentes estrategias. Su certificación, como un resultado de aprendizaje más, pasa por definir la forma en que se van a evaluar. Las competencias transversales toman más relevancia en los procesos de acreditación de la calidad de las titulaciones, en sellos internacionales. Es interesante también tener en cuenta referencias sobre la enseñanza basada en competencias (De Miguel, 2006) (Villa y Poblete, 2007).

A nivel particular de cada asignatura pueden proponerse diferentes actividades formativas que colaboren en el desarrollo de la competencia (actividades grupales, exposición oral, proyectos, trabajos, etc.) que deben tener mecanismos de evaluación acordes con la competencia (prácticas, proyectos, cuestionarios, etc.).

La competencia transversal "Aprendizaje Permanente" es una de las 13 competencias transversales que la Universitat Politécnica de Valencia pretende acreditar (UPV, 2016). Esta competencia se define como "Utilizar el aprendizaje de manera estratégica, autónoma y flexible, a lo largo de toda la vida, en función del objetivo perseguido". Un punto fundamental de esta competencia es el no conformarse con reproducir de manera rutinaria soluciones ya conocidas, sino generar nuevas soluciones o soluciones adaptadas a nuevas situaciones (UPV, 2016). Es una competencia transversal compleja de implementar con menor tradición en evaluarse, que requiere una estrategia distinta en función de la asignatura donde se aplique.

En este trabajo se resume la experiencia de la evaluación de esta competencia transversal en la asignatura "Transductores e Instrumentación Acústica" durante los cursos 2015-2016 y 2016-2017. En el curso 2015-2016 la asignatura pasó a ser punto de control de dicha competencia y se presentaron diferentes iniciativas para su evaluación, planteando actividades para trabajarla, y recogiendo evidencias de los logros alcanzados. Resultados al respecto se presentaron en el congreso IN-RED 2016 (Alba y Del Rey, 2016). En 20162017 se han realizado algunos cambios, en base al DAFO que se planteó en el citado trabajo.

\section{Objetivos}

En la Escuela Politécnica Superior de Gandía (EPSG), en el Grado en Ingeniería de Sistemas de Telecomunicación Sonido e Imagen (GISTSI) se ubica la asignatura "Transductores e Instrumentación Acústica" (TIA) de tercer curso (quinto semestre) en la 
que se ha trabajado durante dos cursos académicos (2015-2016 y 2016- 2017) la competencia transversal "Aprendizaje Permanente", siendo esta asignatura punto de control. En el curso 2015-2016 se incorporó esta competencia transversal y mecanismos para poder evaluarla, y se hizo un análisis DAFO (Alba y Del Rey, 2016). En base al análisis de lo desarrollado en 2015-2016 se plantean modificaciones que se han aplicado en 2016-2017.

Son varios los actos evaluativos que aparecen en la guía docente de la asignatura, que se detallan en el apartado siguiente. La idea fundamental de los cambios aplicados es buscar en cada acto evaluativo indicios de esta competencia transversal. La UPV usa una escala que se utiliza de forma normalizada con los siguientes saltos: A-Excelente, B-Adecuado, CEn Desarrollo y D-No alcanzada. Por tanto, además de buscar estos indicios, han de poder clasificarse o categorizarse para poder obtener una valoración final.

Hay que tener en cuenta que, en general, las competencias transversales no parecen fáciles de evaluar. En el caso concreto del "Aprendizaje Permanente" que se define en la UPV como "utilizar el aprendizaje de manera estratégica, autónoma y flexible, a lo largo de toda la vida, en función del objetivo perseguido" aún parece más complicada. Sin embargo, en la asignatura "Transductores e Instrumentación Acústica", están claros los objetivos perseguidos respecto a sistemas electroacústicos y conocimientos y aplicabilidad de la instrumentación en el campo de la acústica, lo que puede facilitar la búsqueda de indicios.

\section{Desarrollo de la innovación}

La asignatura "Transductores e Instrumentación Acústica" se ubica en tercer curso del GISTSI con 4,5 créditos ECTS distribuidos en 2,5 créditos para teoría de aula, 1 crédito en práctica de aula y 1 crédito de práctica de laboratorio. Es una asignatura optativa de la materia "Sonido e Imagen", que se ubica en tercero y optativa para alumnos de cuarto. Para el curso 2015-2016 sólo se tenía un grupo para teoría de aula y práctica de aula, y dos grupos para práctica de laboratorio. Los recortes de recursos han hecho que en el curso 2016-2017 se eliminara el segundo grupo de prácticas, reduciendo así la capacidad docente y aumentando el tamaño del grupo.

La planificación seguida este último curso 2016-2017 se muestra en la tabla 1. En (Alba y Del Rey, 2016) puede verse la del curso anterior. En la tabla 1 se observa la carga semanal del alumno y los diferentes actos evaluativos definidos. En la tabla 2 se muestra un resumen de los porcentajes de evaluación. Se ha seguido durante los dos cursos el mismo procedimiento.

(cc)) BY-NC-ND 2017, Universitat Politècnica de València 
Tabla 1. Planificación TIA 2016-2017

\begin{tabular}{|c|c|c|c|c|c|}
\hline Fecha & Día & Hora & RECINTO & CONTENIDO & EVALUACION \\
\hline $05 / 09 / 2016$ & $\mathrm{~L}$ & $10: 45$ & AULA 18 & $\begin{array}{l}\text { PRESENTACION. } \\
\text { TEMA } 1\end{array}$ & \\
\hline $07 / 09 / 2016$ & $X$ & $12: 15$ & AULA 18 & TEMA 1 & \\
\hline $12 / 09 / 2016$ & $\mathrm{~L}$ & $10: 45$ & AULA 18 & TEMA 1 & \\
\hline $14 / 09 / 2016$ & $\mathrm{X}$ & $12: 15$ & AULA 18 & TEMA 1 & \\
\hline $19 / 09 / 2016$ & $\mathrm{~L}$ & $10: 45$ & AULA 18 & TEMA 1 & \\
\hline $21 / 09 / 2016$ & $X$ & $12: 15$ & AULA 18 & TEMA 1 & PRUEBA ESCRITA 1 \\
\hline $26 / 09 / 2016$ & $\mathrm{~L}$ & $10: 45$ & AULA 18 & TEMA 2 & \\
\hline $28 / 09 / 2016$ & $X$ & $12: 15$ & AULA 18 & TEMA 2 & \\
\hline $05 / 10 / 2016$ & $X$ & $12: 15$ & AULA 18 & TEMA 2 & \\
\hline $10 / 10 / 2016$ & $\mathrm{~L}$ & $10: 45$ & AULA 18 & TEMA 2 & PRUEBA ESCRITA 2 \\
\hline $17 / 10 / 2016$ & $\mathrm{~L}$ & $10: 45$ & AULA 18 & TEMA 2 & \\
\hline $19 / 10 / 2016$ & $\mathbf{X}$ & 12:15 & B009 & PRACTICA 1 & \\
\hline $24 / 10 / 2016$ & $\mathrm{~L}$ & $10: 45$ & AULA 18 & TEMA 2 & PRUEBA ESCRITA 3 \\
\hline $26 / 10 / 2016$ & $\mathbf{X}$ & $12: 15$ & B004 & PRACTICA 2 & $\begin{array}{l}\text { Entrega práctica } 1 . \\
\text { Prueba minuto } 1 .\end{array}$ \\
\hline $02 / 11 / 2016$ & $\mathrm{X}$ & $12: 15$ & AULA 18 & TEMA 3 & \\
\hline $07 / 11 / 2016$ & $\mathrm{~L}$ & $10: 45$ & AULA 18 & TEMA 3 & \\
\hline $09 / 11 / 2016$ & $\mathbf{X}$ & 12:15 & B009 & PRACTICA 3 & $\begin{array}{l}\text { Entrega práctica } 2 . \\
\text { Prueba minuto } 2\end{array}$ \\
\hline $14 / 11 / 2016$ & $\mathrm{~L}$ & $10: 45$ & AULA 18 & TEMA 3 & PRUEBA ESCRITA 4 \\
\hline $16 / 11 / 2016$ & $\mathrm{X}$ & $12: 15$ & AULA 18 & TEMA 4 & \\
\hline $21 / 11 / 2016$ & $\mathrm{~L}$ & $10: 45$ & AULA 18 & TEMA 4 & \\
\hline $23 / 11 / 2016$ & $\mathbf{X}$ & 12:15 & B009 & PRACTICA 4 & $\begin{array}{l}\text { Entrega práctica } 3 . \\
\text { Prueba minuto } 3 .\end{array}$ \\
\hline $28 / 11 / 2016$ & $\mathrm{~L}$ & $10: 45$ & AULA 18 & TEMA 4 & PRUEBA ESCRITA 5 \\
\hline $30 / 11 / 2016$ & $\mathbf{X}$ & $12: 15$ & B009 & PRACTICA 5/PROYECTO & $\begin{array}{l}\text { Entrega práctica } 4 . \\
\text { Prueba minuto } 4 .\end{array}$ \\
\hline $05 / 12 / 2016$ & $\mathrm{~L}$ & $10: 45$ & B009 & TEMA 5/PROYECTO & PRUEBA ESCRITA 6 \\
\hline $07 / 12 / 2016$ & $\mathrm{X}$ & $12: 15$ & B009 & PROYECTO/TEMA 5 & \\
\hline $12 / 12 / 2016$ & $\mathrm{~L}$ & $10: 45$ & B009 & PROYECTO/TEMA 5 & \\
\hline $14 / 12 / 2016$ & $\mathrm{X}$ & $12: 15$ & B009 & PROYECTO/TEMA 5 & \\
\hline $19 / 12 / 2016$ & $\mathrm{~L}$ & $10: 45$ & B009 & PROYECTO/TEMA 5 & \\
\hline $21 / 12 / 2016$ & $\mathrm{X}$ & $12: 15$ & B009 & PROYECTO/TEMA 5 & $\begin{array}{l}\text { Entrega práctica } 5 . \\
\text { Prueba minuto } 5 . \\
\text { PROYECTO }\end{array}$ \\
\hline
\end{tabular}


Tabla 2. Resumen de la evaluación TIA

\begin{tabular}{|l|l|l|l|}
\hline Tipo & Descripción & Actos & Peso \\
\hline $\begin{array}{l}\text { Pruebas escritas } \\
\text { de respuestas } \\
\text { abierta }\end{array}$ & $\begin{array}{l}\text { Prueba cronometrada, efectuada bajo control, en la } \\
\text { que el alumno construye su respuesta. (Clásico } \\
\text { problema). }\end{array}$ & 5 & $30 \%$ \\
\hline $\begin{array}{l}\text { Evaluación de } \\
\text { prácticas }\end{array}$ & $\begin{array}{l}\text { Informe de cada práctica (informe redactado) y } \\
\text { prueba del minuto. }\end{array}$ & 5 & $25 \%$ \\
\hline Proyecto/PBL & $\begin{array}{l}\text { Los estudiantes por grupos desarrollan un producto } \\
\text { nuevo y propio. }\end{array}$ & 1 & $35 \%$ \\
\hline Coevaluación & Coevaluación unos grupos a otros & 1 & $5 \%$ \\
\hline Autoevaluación & Autoevaluación del grupo y personal. & 1 & $5 \%$ \\
\hline
\end{tabular}

En el curso 2016-2017 se unifican y se simplifican las rúbricas de evaluación entre pares, ya que como se evidenció en el curso anterior, las primeras versiones eran bastante complejas y a los alumnos les costaba interpretarlas (Alba y Del Rey, 2016). En la tabla 4 se muestra la rúbrica de autoevaluación del grupo. En la tabla 5 se muestra la rúbrica de autovaloración individual y por último, en la tabla 6 se muestra la rúbrica de valoración del resto de compañeros de un grupo. Estás últimas se han mantenido en casi su totalidad respecto al curso anterior.

Tabla 3. Rúbrica de evaluación entre pares para presentación oral

\begin{tabular}{|l|l|l|l|l|l|l|}
\hline $\begin{array}{l}\text { Competencias } \\
\text { (1-Malo 2-Regular } \\
\text { 3-Bueno 4-Excelente) }\end{array}$ & GR-1 & GR-2 & GR-3 & GR-4 & GR-5 & GR-6 \\
\hline $\begin{array}{l}\text { 1) Es capaz de diseñar el } \\
\text { equipo/objeto }\end{array}$ & & & & & & \\
\hline $\begin{array}{l}\text { 2) Define los objetivos y las } \\
\text { variables del trabajo }\end{array}$ & & & & & & \\
\hline $\begin{array}{l}\text { 3) Aplicación de criterios } \\
\text { ingenieriles }\end{array}$ & & & & & & \\
\hline $\begin{array}{l}\text { 4) Define los objetivos y las } \\
\text { variables de decisión de su } \\
\text { proyecto }\end{array}$ & & & & & & \\
\hline 5) Resolución del problema & & & & & & \\
\hline 6) Mecánica de la presentación & & & & & & \\
\hline 7) Material de presentación & & & & & & \\
\hline $\begin{array}{l}\text { NOTA GLOBAL PROPUESTA } \\
\text { (0 A 10) }\end{array}$ & & & & & & \\
\hline
\end{tabular}

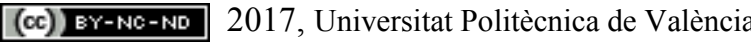


Tabla 4. Rúbrica Autoevaluación 1. Cada miembro del grupo evalua a todo el grupo pensando que funcionan como un equipo

\begin{tabular}{|c|c|c|c|c|c|}
\hline Criterios & $\begin{array}{l}\text { EXCELENTE } \\
(4)\end{array}$ & $\begin{array}{l}\text { BIEN } \\
(3)\end{array}$ & $\begin{array}{l}\text { REGULAR } \\
(2)\end{array}$ & $\begin{array}{l}\text { FLOJO } \\
(1)\end{array}$ & $(1-4)$ \\
\hline $\begin{array}{l}\text { Participación } \\
\text { GRUPAL }\end{array}$ & $\begin{array}{l}\text { Todos hemos } \\
\text { participado con } \\
\text { entusiasmo }\end{array}$ & $\begin{array}{lr}\text { Al menos } & \text { el } \\
75 \% & \text { ha } \\
\text { participado } & \\
\text { activamente } & \end{array}$ & $\begin{array}{l}\text { Al menos la } \\
\text { mitad presentan } \\
\text { ideas propias }\end{array}$ & $\begin{array}{l}\text { Sólo una } \\
\text { persona } \\
\text { participa } \\
\text { activamente }\end{array}$ & \\
\hline $\begin{array}{l}\text { Responsabilidad } \\
\text { COMPARTIDA }\end{array}$ & $\begin{array}{l}\text { Todos } \\
\text { compartimos } \\
\text { por igual la } \\
\text { responsabilidad }\end{array}$ & $\begin{array}{l}\text { La mayor parte } \\
\text { de los miembros } \\
\text { comparten la } \\
\text { responsabilidad }\end{array}$ & $\begin{array}{l}\text { La } \\
\text { responsabilidad } \\
\text { es compartida } \\
\text { por la mitad de } \\
\text { los miembros }\end{array}$ & $\begin{array}{l}\text { La } \\
\text { responsabilidad } \\
\text { recae en una } \\
\text { sola persona }\end{array}$ & \\
\hline $\begin{array}{l}\text { Calidad de la } \\
\text { INTERACCION }\end{array}$ & $\begin{array}{lr}\text { Habilidades } & \text { de } \\
\text { liderazgo } & y \\
\text { saber escuchar; } & \\
\text { conciencia de } \\
\text { los puntos de } \\
\text { vista } \\
\text { opiniones de los } \\
\text { demás }\end{array}$ & $\begin{array}{l}\text { Se conducen } \\
\text { animadas } \\
\text { discusiones } \\
\text { centradas en la } \\
\text { tarea }\end{array}$ & $\begin{array}{lr}\begin{array}{l}\text { Alguna } \\
\text { habilidad }\end{array} \\
\text { interacción: } & \text { se } \\
\text { escucha } & \text { con } \\
\text { atención. } & \\
\text { Alguna } & \\
\text { evidencia de } & \text { discusión } \\
\text { planteamiento } \\
\text { de alternativas }\end{array}$ & $\begin{array}{l}\text { Muy poca } \\
\text { interacción: } \\
\text { conversación } \\
\text { muy breve, } \\
\text { algunos } \\
\text { compañeros } \\
\text { distraídos o } \\
\text { desinteresados }\end{array}$ & \\
\hline $\begin{array}{l}\text { ROLES dentro } \\
\text { del grupo }\end{array}$ & $\begin{array}{l}\text { Cada miembro } \\
\text { tiene un rol } \\
\text { definido: } \\
\text { desempeño } \\
\text { efectivo de roles }\end{array}$ & $\begin{array}{l}\text { Cada miembro } \\
\text { tiene un rol } \\
\text { asignado pero } \\
\text { no } \\
\text { claramente } \\
\text { definido o no es } \\
\text { consistente }\end{array}$ & $\begin{array}{l}\text { Hay roles } \\
\text { asignados pero } \\
\text { no se adhieren } \\
\text { consistentement } \\
\text { e a ellos }\end{array}$ & $\begin{array}{l}\text { No hay ningún } \\
\text { esfuerzo de } \\
\text { asignar roles }\end{array}$ & \\
\hline
\end{tabular}

Tabla 5. Rúbrica Autoevaluación 2: cada miembro se autoevalua -autocalifica la participacion el proyecto (mi autoevaluación) Puntos: 0-nada, 1-poco, 2-suficiente, 3 - mucho

\begin{tabular}{|c|c|c|c|}
\hline CRITERIO & $\begin{array}{l}\text { PUNTOS } \\
(0-3)\end{array}$ & CRITERIO & $\begin{array}{l}\text { PUNTOS } \\
(0-3)\end{array}$ \\
\hline a. Respeto turnos o tiempo & & j. Soy paciente & \\
\hline $\begin{array}{l}\text { b. Intervengo en el contexto del } \\
\text { trabajo cuando creo necesario }\end{array}$ & & k. Mostré conocimientos previos & \\
\hline c. Aporto sugerencias & & $\begin{array}{lcr}\text { l. Poseo } & \text { habilidad } & \text { para } \\
\text { determinar } & \text { alternativas } & \text { de } \\
\text { solución frente a problemas } & \\
\end{array}$ & \\
\hline d. Apoyo otras sugerencias & & $\begin{array}{l}\text { m. Considero consecuencias al } \\
\text { momento de decidir sobre cómo } \\
\text { aporto al trabajo }\end{array}$ & \\
\hline e. Apoyo otras opiniones & & n. Identifico las oportunidades & \\
\hline f. Aporté con temas al grupo & & ñ. Tengo facilidad para tomar & \\
\hline
\end{tabular}




\begin{tabular}{|c|c|}
\hline & decisiones \\
\hline g. Opino razonadamente & o. Mi nivel de aportación es... \\
\hline h. Acepto ideas & p. Cumplo tareas \\
\hline $\begin{array}{l}\text { i. Favorezco las actitudes } \\
\text { integradoras }\end{array}$ & $\begin{array}{l}\text { q. Mis tareas al interior del grupo } \\
\text { fueron terminadas }\end{array}$ \\
\hline
\end{tabular}

Tabla 6. Rúbrica 3 autoevaluación. Cada miembro del grupo evalua al resto de sus compañeros.

Hay una tabla para cada compañero

\begin{tabular}{|c|c|c|c|c|c|}
\hline Criterios & EXCELENTE (4) & BIEN (3) & REGULAR (2) & FLOJO (1) & $P(1-4)$ \\
\hline $\begin{array}{l}\text { Interactividad } \\
\text { uno a uno }\end{array}$ & $\begin{array}{lr}\text { Parafrasea } & \text { para } \\
\text { mostrar } & \text { la } \\
\text { comprensión. } & \\
\text { Aprende de } & \text { los } \\
\text { demás. } & \text { Da } \\
\text { retroalimentación } \\
\text { positiva. } \\
\text { sugerencias. }\end{array}$ & $\begin{array}{l}\mathrm{Da} \\
\text { retroalimentación } \\
\text { positivar } \quad \mathrm{Se} \\
\text { muestra algo } \\
\text { interesado. }\end{array}$ & $\begin{array}{l}\text { Hace saber a sus } \\
\text { compañeros que lee } \\
\text { los mensajes }\end{array}$ & $\begin{array}{lr}\text { Sigue } & \text { el } \\
\text { proceso } & \text { del } \\
\text { grupo } & \end{array}$ & \\
\hline Contribución & $\begin{array}{l}\text { Genera nuevas ideas } \\
\text { con base en un } \\
\text { análisis cuidadoso } \\
\text { del proceso y de los } \\
\text { recursos }\end{array}$ & $\begin{array}{l}\text { Fundamenta las } \\
\text { ideas del grupo } \\
\text { usando múltiples } \\
\text { fuentes }\end{array}$ & $\begin{array}{l}\text { Muestra información } \\
\text { relevante de fuentes } \\
\text { de información } \\
\text { válidas } \\
\text { identificadas }\end{array}$ & $\begin{array}{l}\text { Envía datos } \\
\text { o } \\
\text { información }\end{array}$ & \\
\hline $\begin{array}{l}\text { Responsabilid } \\
\text { ad }\end{array}$ & $\begin{array}{l}\text { Ayuda a organizar el } \\
\text { trabajo y planea } \\
\text { contactos. Ayuda a } \\
\text { todos a cumplir con } \\
\text { sus compromisos }\end{array}$ & $\begin{array}{l}\text { Planea el trabajo } \\
\text { con otros } \\
\text { miembros. } \\
\text { Comparte su plan } \\
\text { individual. }\end{array}$ & $\begin{array}{l}\text { Es confiable, entrega } \\
\text { la información en los } \\
\text { tiempos convenidos }\end{array}$ & $\begin{array}{l}\text { Entrega la } \\
\text { información } \\
\text { pero fuera } \\
\text { de los } \\
\text { tiempos } \\
\text { convenidos }\end{array}$ & \\
\hline $\begin{array}{l}\text { Discusión } \\
\text { para la } \\
\text { construcción } \\
\text { del } \\
\text { conocimiento }\end{array}$ & $\begin{array}{l}\text { Muestra acuerdos y } \\
\text { desacuerdos. Sirve } \\
\text { de mediador para } \\
\text { hacer que las ideas } \\
\text { avancen }\end{array}$ & $\begin{array}{l}\text { Construye ideas, } \\
\text { resume, sintetiza }\end{array}$ & $\begin{array}{l}\text { Anima a los demás, } \\
\text { parafrasea ideas, } \\
\text { mantiene la } \\
\text { conversación } \\
\text { enfocada en el } \\
\text { problema, construye } \\
\text { argumentos lógicos. }\end{array}$ & $\begin{array}{lr}\text { Hace } & \text { saber } \\
\text { a r sus } \\
\text { compañeros } \\
\text { que está } \\
\text { leyendo sus } \\
\text { mensajes }\end{array}$ & \\
\hline
\end{tabular}

La competencia transversal de "aprendizaje permanente" se va evaluando en base a indicadores que se definen en las pruebas evaluativas (tabla 2), teniendo en cuenta la escala A-Excelente, B-Adecuado, C-En Desarrollo y D-No alcanzada:

- Pruebas escritas: se revisan los materiales que usan los alumnos cuando la resuelven valorando si se limitan a cumplir los mínimos o han buscado materiales alternativos. No traer nada se califica "D", limitarse a traer lo aportado por el profesor se califica " $\mathrm{C}$ " o " $\mathrm{B}$ " en función de si el material está trabajado y traer nuevo material relevante para la prueba escrita se califica " $\mathrm{A}$ ".

- Memorias de prácticas: han de hacer referencia a normativas, leyes y procedimientos en las que se basa, viendo el grado de profundización. No aportar

(cc) EY-NC-ND 2017, Universitat Politècnica de València

Congreso In-Red (2017) 
ninguna referencia se califica " $D$ ". Aportar alguna referencia pero incompleta $u$ obsoleta se califica "C". "B" para el caso en que hayan referencias actualizadas y "A" en el caso de revisar normativas o referencias alternativas.

- Pregunta del minuto: se relaciona directamente con el aprendizaje permanente. En el desarrollo de cada práctica se citan enlaces y referencias. Por ejemplo, en el caso de medidas de directividad se cita a la Audio Engineering Society, muy relacionada con este tipo de medidas. En la pregunta del minuto se pregunta sobre el parámetro de medida y su unidad. Quien ha tenido interés contesta hablando de lo que la AES recomienda. El que no se ha preocupado, contesta simplemente lo medido con la memoria de prácticas. Las preguntas evolucionan hasta el punto de dar diferentes referencias y formas de búsqueda que permiten más de una contestación válida, pero que requiere profundizar en el tema. No contestar se califica "D". Contestar en base a lo medido se califica como "C" o "B" en función del grado de detalle y claridad. Aportar alguna magnitud adicional no explicada en la práctica se califica como “A.

- PBL: en principio el proyecto se puede definir de forma abierta. El grado de autonomía y estrategia seguido para la realización es lo que se tiene en cuenta. Se puede proponer un trabajo por parte del alumno. En función del grado de detalle, su grado de resolución y las rúbricas, se califica " $A$ " o "B". En el caso del trabajo proporcionado por el profesor se califica "B" o " $\mathrm{C}$ " en función del grado de realización y rubricas. En casos excepcionales como "A" si aporta algo nuevo no contemplado en el diseño original. Proyectos inacabados o con resolución inapropiada se califican como " $\mathrm{D}$ ".

Como hay que aportar una única valoración desde "A" hasta " $D$ ", se valora una evaluación progresiva. Se ponderan con más peso las últimas entregas, y en función del porcentaje de ponderación de las pruebas. Se ordinalizan las letras de $D=1$ hasta $A=4$. Se obtiene un valor numérico entre 1 y 4 que se redondea al valor entero más cercano y se le reasigna la letra de la calificación final.

\section{Resultados}

En el curso 2015-2016 hubo 21 alumnos matriculados y 18 en el curso 2016-2017. Se ha partido del planing de la tabla 1 en la organización para el curso 2016-2017 (y hubo uno similar adaptando fechas para el curso anterior). Este planing no ha sufrido cambios apreciables. En los dos cursos ha pasado lo mismo. Hasta mediados de noviembre todos los alumnos han seguido el ritmo planificado. En la primera semana de noviembre ya estaban los trabajos elegidos y los grupos distribuidos. Los actos evaluativos han seguido la tabla 2. Se pueden realizar los siguientes comentarios:

- 5 pruebas escritas individuales, con los apuntes delante: Los alumnos las han resuelto sin dificultad.

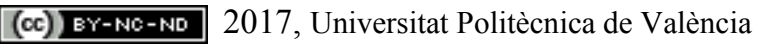


- 5 memorias de prácticas grupales: se producen retrasos y caída de la calidad de la memoria en las entregas finales, a partir de finales de noviembre. La mayoría de grupos opta a mejorar nota de prácticas.

- 5 preguntas del minuto asociadas a las prácticas individuales: se han detectado anomalías de grupo que luego se han visto en las rúbricas de evaluación entre pares y autoevaluación.

- Proyecto grupal de la asignatura. Estos dos cursos han realizado diferentes trabajos: bocinas de exteriores, software de altavoces, fabricación de cajas, construcción de un altavoz dinámico de bajo coste, construcción de un bass-reflex, construcción de un line-array, construcción de una unidad Leslie Tremolo, Medidas de las vibraciones del aire acondicionado. A continuación se muestran algunas fotografías del curso 2016-2017 (figura 1):
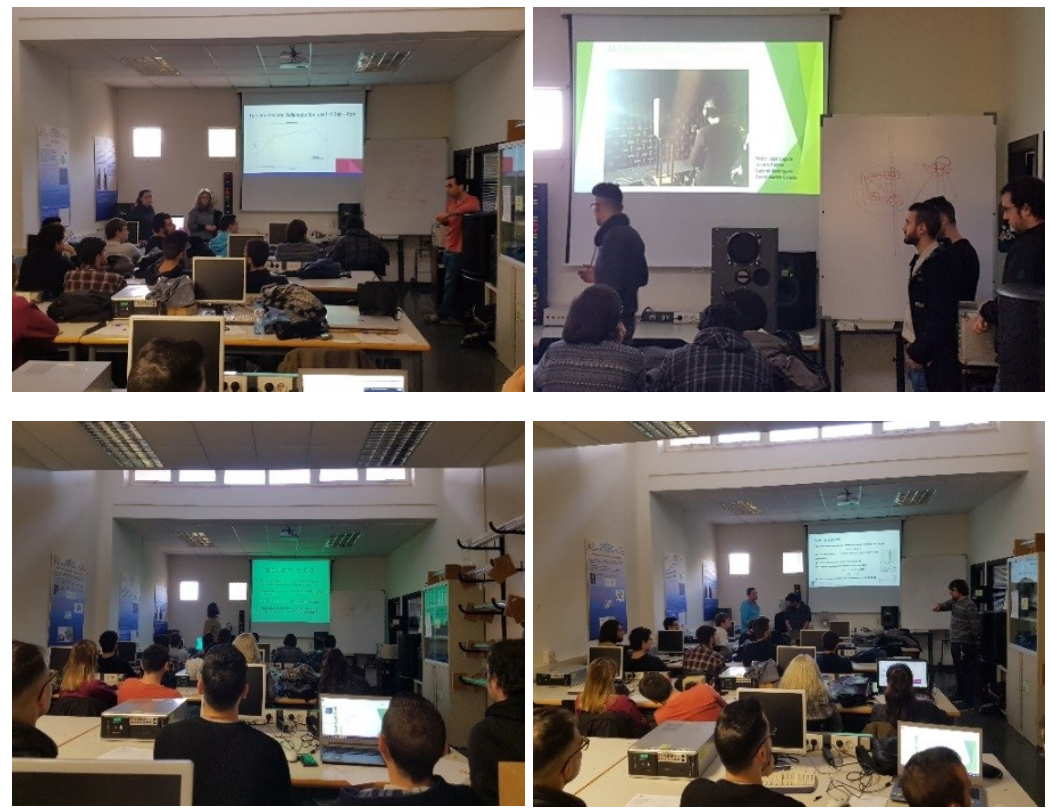

Fig. 1 Imágenes de una clase durante el curso 2016-2017.

Los trabajos se entregan en enero pero la presentación del trabajo se realiza antes de las vacaciones de Navidad. Esto obliga a los grupos a presentar lo que tienen preparado hasta la fecha. Este formato es una novedad para los alumnos, pero sirve para saber realmente si están avanzando en su proyecto, si saben lo que están haciendo, y si son capaces de defender su trabajo. Para ello disponen de las siguientes rúbricas:

- Rúbrica de evaluación entre pares del trabajo: El día de la presentación los alumnos realizan una rúbrica de todos los grupos menos el suyo (tabla 3). La mayoría de los resultados se califican de "excelente" aunque se evidencia que algún grupo aún no ha avanzado nada en su proyecto. El profesor también realiza la rúbrica en la misma tabla. Realmente se ven diferencias en la nota global propuesta y no tanto en cada ítem.

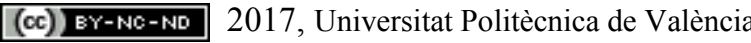


- Rúbricas de autoevaluación de los grupos (prácticas y trabajo). A la entrega del trabajo también entregan las rubricas de las tablas 4, 5 y 6 . Los datos generales son casi siembre un 4 en la valoración del grupo y de sus compañeros. La autoevaluación entre 2 y 3 casi siempre, excepto algún caso de problema en un grupo.

En estos dos últimos cursos todos los alumnos han superado la asignatura. En el diagrama siguiente (figura 2) puede verse la distribución en porcentaje de la valoración del curso 2016-2017 en funcion de los siguientes ítems: A-Excelente, B-Adecuado, C-En Desarrollo y D-No alcanzada.

\section{CT-11. Aprendizaje permanente}

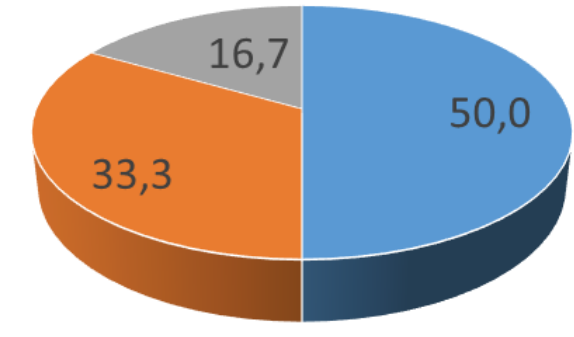

$\square \mathrm{C} \square \mathrm{B}=\mathrm{A}$

Fig 2. Diagrama de valoración de la competencia transversal “Aprendizaje Permanente” durante el curso 2016-2017.

La mitad de los alumnos tienen la competencia en desarrollo, un tercio se puede considerar como adecuado y el resto han mostrado una buena capacidad para el aprendizaje permanente. Esto es resultado de aplicar los criterios descritos en el desarrollo de la innovación. La mitad de los alumnos se están limitando a realizar los procesos que le indica el profesor, pero esto no es garantía de aprendizaje permanente. Un tercio han aportado indicios basados en propuestas propias con búsquedas propias y un grado adecuado de aprendizaje permanente. El resto han demostrado gran interés en el aprendizaje permanente, llegando a aportar elementos propios y con un buena parte de las propuestas con alternativas.

\section{Conclusiones}

Se plantea una reflexión en base a un DAFO de estos dos cursos.

Respecto a las fortalezas:

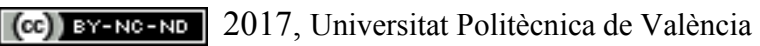


- Realmente existe un seguimiento del alumno durante toda la asignatura con la combinación de varias técnicas, sin necesidad de realizar examen final.

- La pregunta del minuto es una buena herramienta para detectar carencias en prácticas y también de anomalías en el grupo de prácticas.

- La presentación oral intermedia en base a 3-4 transparencias facilita el seguimiento de los trabajos y de los grupos.

- Los alumnos prefieren que no haya examen en la asignatura

- Los pequeños actos evaluativos hacen que el alumno siga la asignatura y se obligue a estar al día.

- La segunda revisión de memorias de prácticas facilita la comprensión y la asimilación de conceptos por parte de los alumnos. También ayuda a distinguir qué alumnos tienen mayor interés.

Las debilidades detectadas:

- La carga de trabajo de alumnos y profesores es mucho mayor que en otras asignaturas del mismo semestre, sobre todo en el primer curso de implantación (en el segundo se rebaja algo la carga).

- El ritmo de los trabajos no es fluido cuando se convive con asignaturas de evaluación tradicional en base a exámenes. Esto se observa en la bajada de resultados a partir de mediados de noviembre.

- Las rúbricas de coevaluación no aportan mucha información, si se analiza cada ítem. Sólo hay diferencias en la calificación global de siempre es superior a 7. Debe normalizarse de otra forma.

- Las rúbricas de autoevaluación han servido para detectar problemas entre compañeros de un grupo. Hay casos donde todos los compañeros ponen la calificación más baja a un miembro. Otros casos donde todos tienen calificación baja, menos un miembro. Esto hay que analizarlo con detalle.

Las amenazas de se han detectado estos dos cursos:

- Las asignaturas que siguen una evaluación más clásica, con exámenes finales, interfieren de forma clara. Los alumnos acumulan en las últimas semanas a veces hasta dos exámenes al día de otras asignaturas. Esto rompe el ritmo de los trabajos.

- No existe una apuesta real por este tipo de metodologías. Del curso 2015-2016 al 2016-2017 se eliminó un grupo de prácticas.

Evaluar las competencias transversales es una oportunidad real de poder realizar un cambio en la manera de enfocar la educación en las asignaturas, aumentando su calidad y proporcionando herramientas que ayuden a certificar las titulaciones en el ámbito internacional. Debe revisarse el método de valoración de la competencia transversal para bajar su grado de complejidad evaluativo. 


\section{Referencias}

ALBA J., DEL REY R. (2016) Competencia transversal "Aprendizaje Permanente": experiencia en la asignatura Transductores e Instrumentación Acústica. Universitat Politècnica de València Congreso IN-RED 2016.

ALBA J., DEL REY, R., VIDAL A., ROIG B. (2015a) Aprendizaje Basado en Proyectos en el Grado en Ingeniería de Sistemas de Telecomunicaciones, Sonido e Imagen. Caso práctico del electroscópio como experiencia interdisciplinar entre Física y Matemáticas. 23 Congreso Universitario de Innovación Educativa de las Enseñanzas Técnicas (XXIII CUIEET). Valencia 15-17 de julio de 2015.

ALBA J., TORREGROSA C., DEL REY R. (2015b) Aprendizaje basado en proyectos: Primera experiencia en la asignatura de Física del Grado en Ingeniería de Telecomunicación, Sonido e Imagen. Universitat Politècnica de València Congreso IN-RED (2015)

APARICIO, F., GONZALEZ, R. M. Y SOBREVILA, M. A. (2005). Formación de Ingenieros. Objetivos, métodos y estrategias. Instituto de Ciencias de la Educación, UPM.

CASE, J. M. Y LIGHT, G. (2011). Emerging Methodologies in Engineering Education Research. Journal of Engineering Education, 100 (1), 186-210.

CALVO, I., LOPEZ-GUEDE, J.M. Y ZULUETA, E. (2010). Aplicando la metodología Project Based Learning en la docencia de Ingeniería Técnica en Informática de Gestión, Revista de Formación e Innovación Educativa Universitaria. Vol. 3, No 4, 166-181

DE MIGUEL, M. (2006). Modalidades de Enseñanza centradas en el desarrollo de competencias. Universidad de Oviedo.

GONZÁLEZ, J. y WAGENAAR, R. (2003): Tuning Educational Structures in Europe. Informe Final - Proyecto Piloto, Fase 1, Bilbao, Universidad de Deusto.

UPV, UNIVERSITAT POLITECNICA DE VALENCIA. Competencias transversales UPV $<$ http://competenciast.webs.upv.es/> [Consulta: 1 de abril de 2016]

VILLA, A. Y POBLETE, M. (2007): Aprendizaje basado en competencias. Una propuesta para la evaluación de las competencias genéricas. Ediciones Mensajero. Bilbao. 